\title{
Brown Dwarfs in the Alpha Persei Cluster
}

\author{
John R. Stauffer \\ SIRTF Science Center, California Institute of Technology, Pasadena, \\ CA 91125
}

David Barrado y Navascués

LAEFF-INTA, Apdo. 50727, Madrid 28080 SPAIN

Jerome Bouvier

Observatoire de Grenoble, Université J. Fourier, Grenoble Cedex 9, 38041 France

Nicholas Lodieu

Astrophysikalisches Institut Potsdam, Potsdam, 14482 Germany

Mark McCaughrean

Astrophysikalisches Institut Potsdam, Potsdam, 14482 Germany

\begin{abstract}
We have obtained a new, deep, wide-field optical imaging survey of the young Alpha Persei cluster which reveals a well-populated lower main sequence extending into the substellar mass regime. Subsequent infrared photometry confirms that most of the candidate brown dwarfs are indeed likely to be cluster members, with a predicted minimum mass of order 0.035 solar masses. We have combined the new candidate list with previous member catalogs to derive an IMF for Alpha Per; the slope of the IMF at the low mass end is $\alpha \sim 0.6$. The Alpha Per IMF slope is thus very similar to that found in the Pleiades.
\end{abstract}

\section{Introduction}

To those of us who work on open clusters "for a living", it is obvious why we spend inordinate amounts of time concentrating on just a handful of open clusters. To those of you who do not dream of open clusters at night, it is probably not nearly so obvious why the same clusters keep appearing in the titles of astro-ph listings. Are open cluster specialists simply unimaginative, or are there good reasons for the single-minded pursuit of just a few clusters?

In my opinion, open cluster specialists are simply "dealing with the hand that they were dealt". In particular, the table below shows ALL of the open clusters nearer than $200 \mathrm{pc}$ and younger than $200 \mathrm{Myr}$. Ignoring star-forming regions and co-moving groups like the TW Hya association, the clusters in this 
table are by definition the ones which are the best targets for brown dwarf searches. The next best candidates (e.g. NGC 2516, M34, NGC6475) tend to be both more distant and somewhat older, resulting in the apparent magnitude of the hydrogen burning mass limit being two or more magnitudes fainter than for the clusters of Table 1. You hear so much about the clusters in Table 1 simply because they are the logical choice for the specific science goal.

Table 1. The Nearest, Youngest Open Clusters

\begin{tabular}{lcc}
\hline Cluster & $\begin{array}{c}\text { Distance } \\
(\mathrm{pc})\end{array}$ & $\begin{array}{c}\text { Upper Main Sequence } \\
\text { Age }(\mathrm{Myr})\end{array}$ \\
\hline IC 2391 & 150 & 35 \\
IC 2602 & 150 & 35 \\
Alpha Persei & 170 & 50 \\
Pleiades & 118 & 100
\end{tabular}

Compared to the other clusters in Table 1, Alpha Persei is richer than the two IC clusters but possibly less rich than the Pleiades. Alpha Per has of order 400 cataloged members to date (compared to of order 800 for the Pleiades); however, the Alpha Per member list is much less complete, and a full cluster member list for the two clusters might well be of comparable length. The primary reasons for the less complete member list for Alpha Persei are: (a) the mean proper motion of the cluster is less well separated from the field in a vector-point diagram and (b) Alpha Persei is at much lower galactic latitude $\left(-6^{\circ}\right.$ compared to $-23^{\circ}$ for the Pleiades), greatly increasing the surface density of field stars projected onto the direction of the cluster.

\section{Previous Surveys for Low Mass Members of Alpha Per}

The Pleiades is the poster child for open cluster brown dwarf searches and more generally for the identification and physical study of low mass members. The number of proper motion and/or deep imaging surveys to identify low mass members of the Pleiades easily exceeds twenty, with an equal or larger number of followup papers providing additional data or analysis of the candidate VLM/BD members. As noted above, the competition to identify low mass members of the Alpha Persei cluster has been much less intense. Table 2 below summarizes all of the published papers designed to identify low mass members of Alpha Per, the first two of which barely reach below $0.5 \mathrm{M}_{\odot}$. The two Prosser surveys reach much deeper, but probably do not quite reach the hydrogen burning mass limit.

Table 2. Previous Searches for Low Mass Members of Alpha Per

\begin{tabular}{ccc}
\hline Year & Reference & Properties \\
\hline 1985 & Stauffer et al. ApJ 289, 247 & 1.4 sq. deg. to V $\sim 16$ \\
1989 & Stauffer et al. ApJ 346, 160 & 2.5 sq. deg. to V $\sim 16$ \\
1992 & Prosser AJ 103, 488 & $\sim 20$ sq. deg. to V $\sim 19$ \\
1994 & Prosser AJ 107, 1422 & $\sim 0.8$ sq. deg. to V $\sim 22$ \\
1999 & Stauffer et al. ApJ 527, 219 & $\sim 6$ sq. deg. to I $\sim 19$
\end{tabular}


The first three surveys in Table 2 all incorporated proper motions to help identify the true cluster members. In order to reach to lower masses, however, it was necessary to obtain CCD images that were deeper than existing photographic plate data and for which there were no appropriate first epoch material from which to derive proper motions. These deep imaging surveys therefore necessarily identify candidate members initially only by the location of the candidate in a color magnitude diagram (V vs. V-I or I vs. R-I). The Stauffer et al. 1999 paper identified 20 probable Alpha Per members with $16.5<\mathrm{I}<19$. Spectra of those candidate very-low mass cluster members indicated that five of them had strong $\mathrm{Li} \lambda 6708 \AA$ absorption features, with all of these stars having I > $17.7(\mathrm{M}(\mathrm{I})>11.5)$. Using models from Chabrier \& Baraffe and Burrows, Stauffer et al. concluded that the lithium depletion boundary age of Alpha Persei was $\sim 90 \mathrm{Myr}$. For that age, the two faintest Alpha Per candidates from the 1999 survey have inferred masses below the hydrogen burning mass limit.

\section{New Surveys for Substellar Members of the Alpha Per Cluster}

The survey reported by us in 1999 was relatively wide and shallow, since the goal was to identify as many candidate Alpha Per members as possible with I magnitudes near the expected location of the lithium depletion boundary. We have recently completed analysis of a much deeper but smaller area survey designed to reach well below the lithium depletion boundary (Barrado y Navascués et al. 2002). The optical part of that survey covered about 3 square degrees in $\mathrm{R}$ and I, used the KPNO $4 \mathrm{~m}$ CCD mosaic camera, and was complete to I 19.5. The faintest candidate Alpha Per member had $\mathrm{I} \sim 22$. Figure 1 shows the color-magnitude diagram derived from our images.

Figure 1 shows a fairly good separation between the locus of the Alpha Per members and the great majority of the field stars. However, because of the low galactic latitude of the cluster, we still expected significant contamination by field stars. For that reason, we obtained near-IR photometry for all but two of these candidates, using new data from Calar Alto and publicly available JHK photometry from 2MASS. We constructed a variety of color-color and colormagnitude diagrams from the RIJHK photometry, and eliminated objects whose colors were inconsistent with the Alpha Per locus defined by previously-identified members and by theoretical tracks from Chabrier and Baraffe (Baraffe et al. 1998; Chabrier et al. 2000). Of the 94 candidates identified from the optical photometry, 54 were retained as probable members based on the multicolor criteria, 12 were retained as possible candidates, and 26 were rejected as likely non-members.

Based on our recent experience with using multi-color photometry to identify substellar members of the Pleiades (Martín et al. 2000), we are relatively confident that the list of 54 candidate members is good and contains few spurious objects. However, we thought it still prudent to obtain spectroscopic confirmation of a sample of these stars. We used the Keck Low-Resolution Imaging Spectrograph (LRIS; Oke et al. 1995) for this effort. Poor weather limited the sample size, but we managed to obtain spectra of 10 objects - 7 candidate members from the list of 54 , and three control objects with similar but slightly discrepant colors. The spectra provided good confirmation of our sample se- 


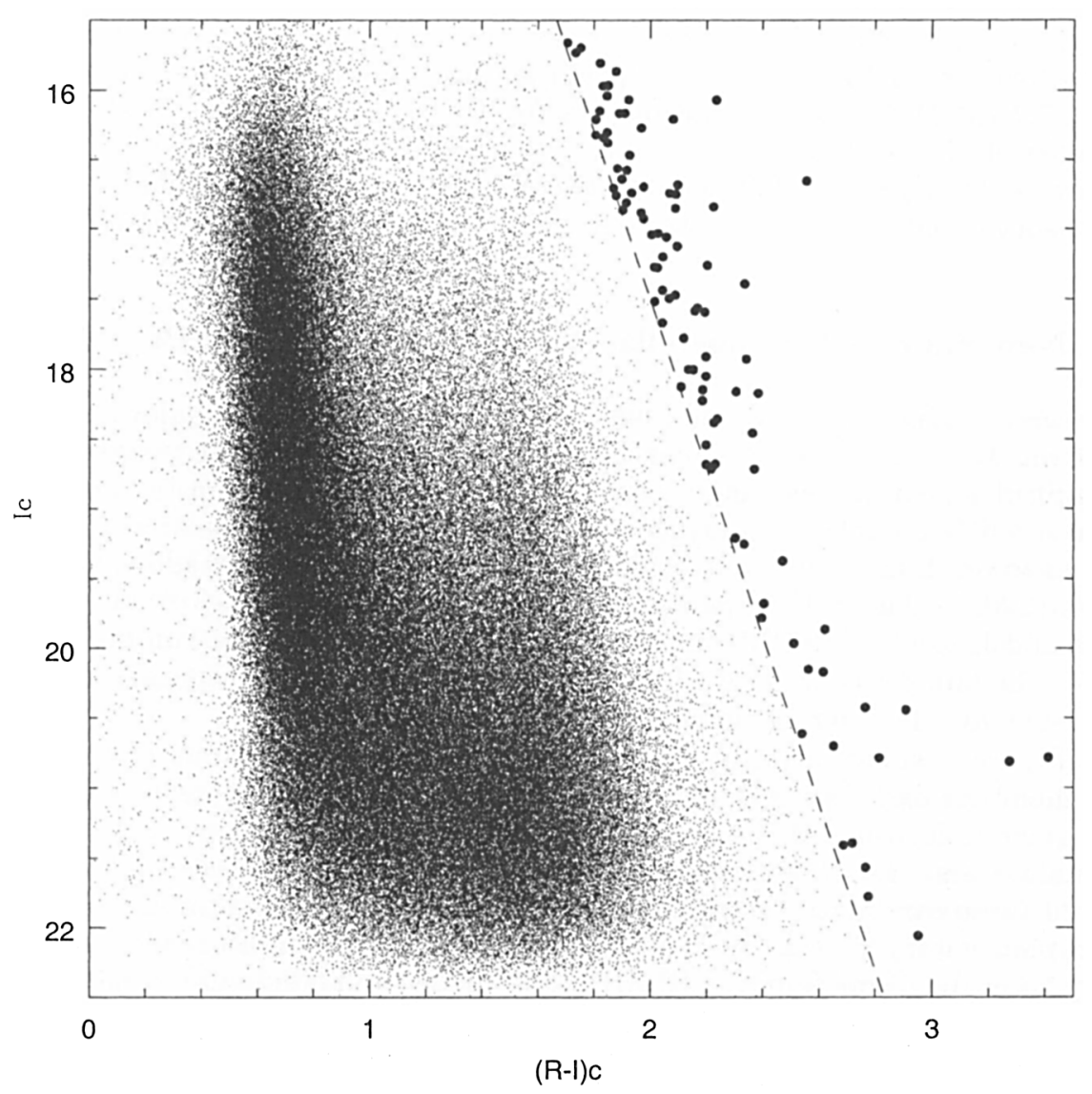

Figure 1. I vs. R-I CMD from our KPNO CCD mosaic survey. Large filled dots are the new candidate Alpha Per cluster members. The dashed line is an empirical lower-envelope for Alpha Per based on photometry of the previously identify VLM/BD cluster members. 


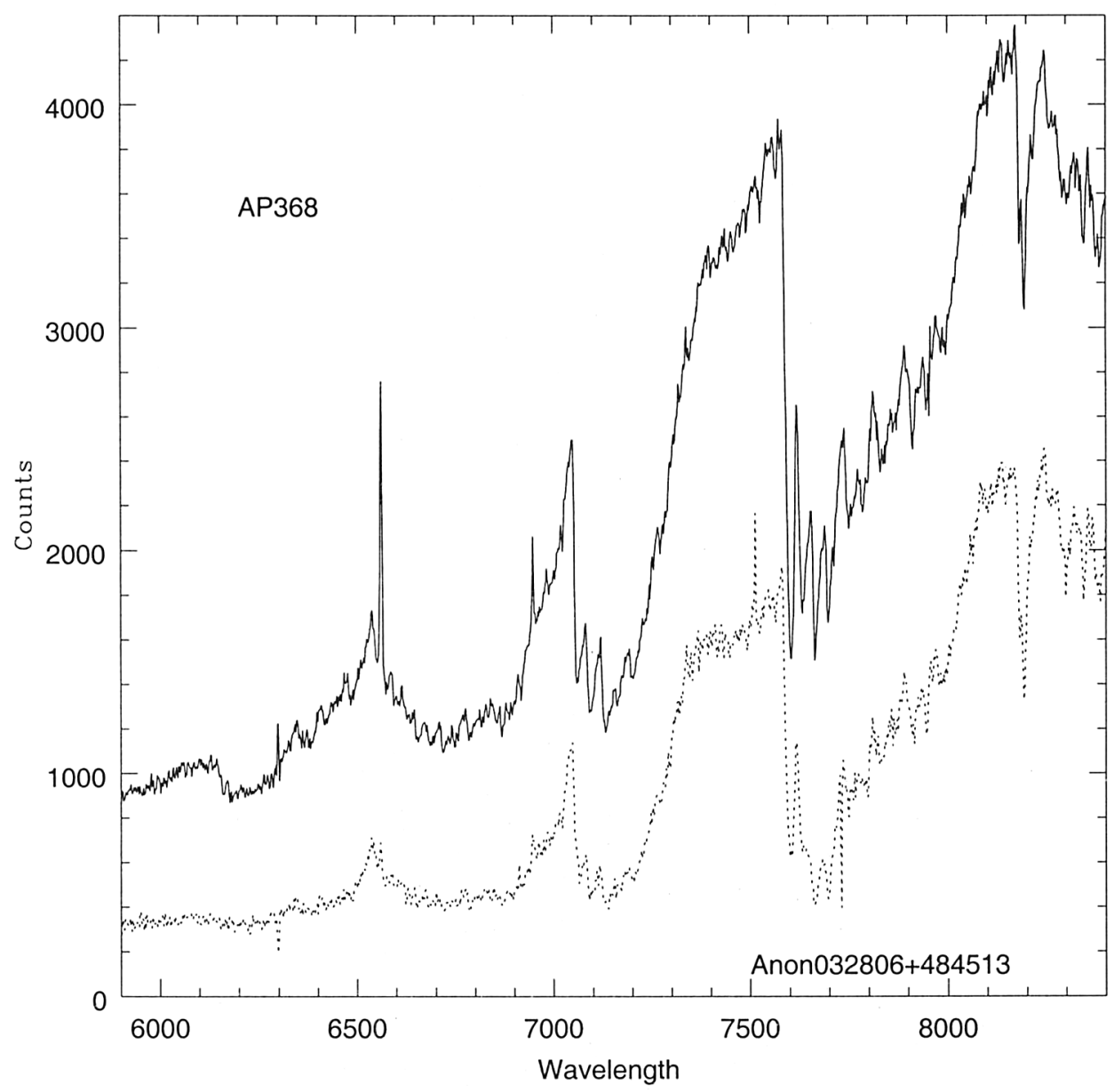

Figure 2. Keck LRIS spectra of one of our new candidate VLM Alpha Per members (AP368) and a control object which we expected to be a field $\mathrm{M}$ dwarf based on its multicolor photometry. For clarity, the spectrum of AP368 has been shifted upwards by adding a constant. 
lection. The seven candidate members all show relatively strong $\mathrm{H} \alpha$ emission (equivalent width $>5 \AA$ ), relatively weak NaI $\lambda 8200 \AA$ absorption (as expected for these somewhat pre-main-sequence stars), and spectral types between M5 and M6 (as expected based on their colors). The three control objects are much more diverse - one is a heavily reddened $\mathrm{K}$ dwarf, another is an $\mathrm{M}$ dwarf with weak or no $\mathrm{H} \alpha$ emission, and the third is an $\mathrm{M}$ dwarf with relatively strong $\mathrm{H} \alpha$ emission but also with stronger $\mathrm{NaI} \lambda 8200 \AA$ than the Alpha Per members (consistent with it being a somewhat older, field $M$ dwarf on the ZAMS). Our conclusion is that the candidate cluster member list derived from the multicolor photometry should have little contamination, and thus should be appropriate for further analysis. Figure 2 shows spectra of one of the candidate cluster members and the probable field $\mathrm{M}$ dwarf that lacked $\mathrm{H} \alpha$ emission.

Our main goal for this study was to identify substellar members of Alpha Per. Based on the Chabrier and Baraffe models and an assumed $90 \mathrm{Myr}$ age for the cluster, 11 of our new candidates should have masses below the hydrogen burning mass limit (I > 18.2). The inferred mass for our faintest candidate at I $\sim 20.8$ is $\sim 0.035 \mathrm{M}_{\odot}$. Assuming the IMF can be functionally characterized as $\mathrm{dN} / \mathrm{dM} \propto \mathrm{M}^{-\alpha}$, our data are consistent with a slope of that relation of $\alpha \sim 0.6$.

Our group has also recently completed a deep near-IR imaging survey of about one square degree of the Alpha Per cluster in order to attempt to identify even lower mass members of the cluster. Details of this program are described elsewhere in these proceedings in the paper by Lodieu et al.

\section{What About the Star Alpha Persei?}

The Alpha Persei cluster derives its name from its brightest member - the star Alpha Persei. Alpha Persei is an F supergiant. In general, it has been assumed to be a member of the cluster based on its spatial location. However, there are reasons to question that conclusion, particularly given the age derived for the cluster from the lithium depletion boundary method of $\sim 90 \mathrm{Myr}$. The problem is that the older generation of theoretical models (without convective core overshoot) required an age for the cluster less than $50 \mathrm{Myr}$ in order for the isochrone to pass near the location of the star Alpha Persei in an HR diagram. That age is clearly inconsistent with the LDB age.

There are two new inputs to this question from recent journal articles. First, the Hipparcos data have allowed a much better determination of the proper motions of the high mass members of the cluster. From an analysis of those data, de Zeeuw et al. (1999) have concluded that Alpha Per the star is indeed a highly probable member of the cluster, with membership probability $\mathrm{P}=$ $97 \%$. Second, several new evolutionary tracks are now available which include convective core overshoot, with the size of the overshoot region constrained by a sizeable database of TAMS binary systems (cf. Andersen 1995, Bertelli 2000). Even with those models (e.g. Meynet, Mermiliod \& Maeder 1993 and Girardi et al. 2002), however, the inferred age for Alpha Persei the star is of order $55 \mathrm{Myr}$, with the $90 \mathrm{Myr}$ isochrone being much too red and too faint to be consistent with the photometric properties of Alpha Per. Figure 3 shows an Alpha Persei HR diagram with comparison to the latest Padua models (Girardi et al. 2002), illustrating this difficulty. 


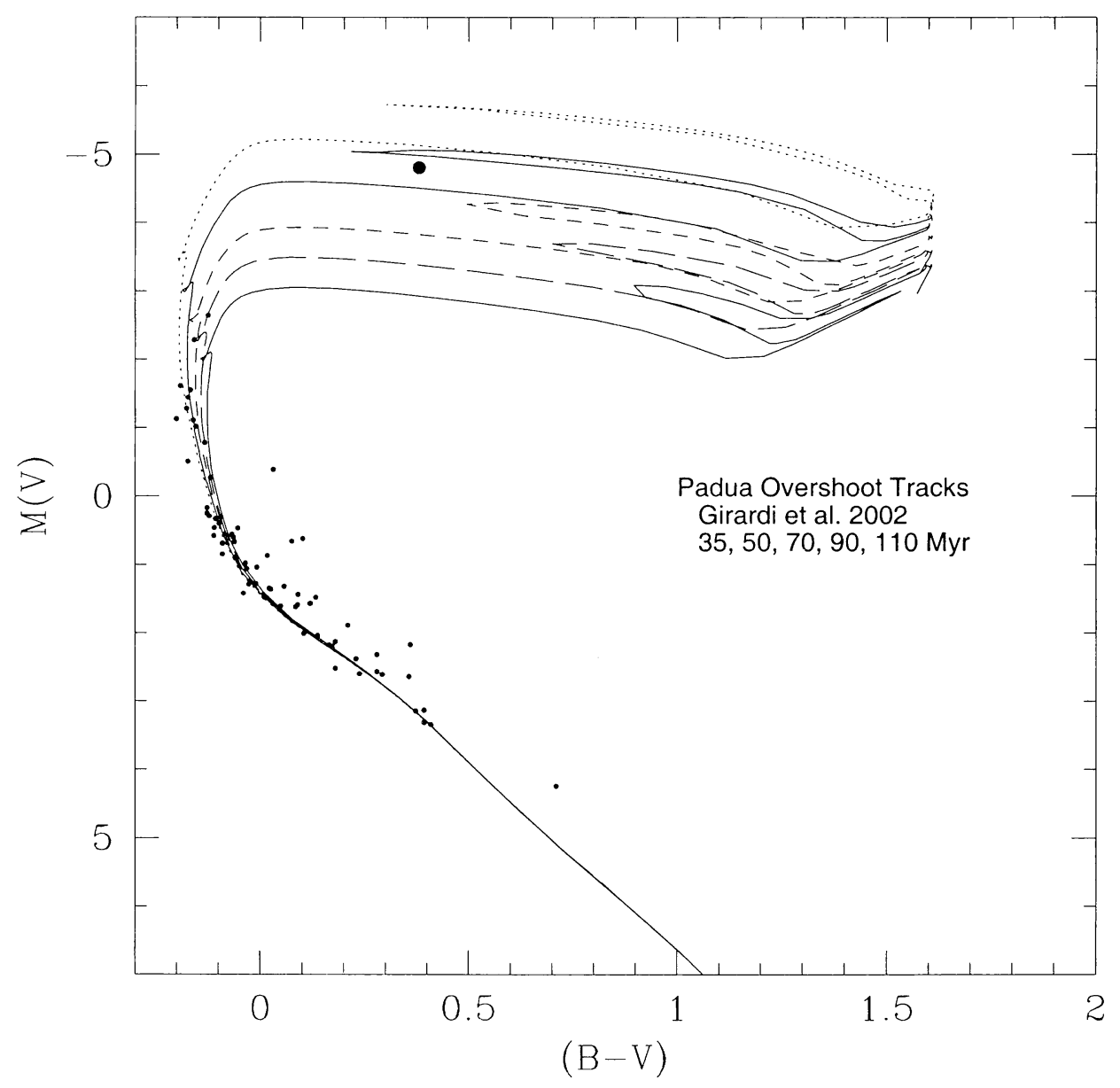

Figure 3. Comparison of photometry of Alpha Per cluster members to one of the most recently published sets of isochrones. The namesake for the cluster - the F supergiant Alpha Persei - is the large filled dot near the top of the diagram. The inferred age for the star Alpha Persei from this diagram is slightly older than $50 \mathrm{Myr}$. 


\section{References}

Andersen, J. 1995, in IAU Symposium 166, 187.

Baraffe, I., Chabrier, G., Allard, F., \& Hauschildt, P. 1998, A\&A, 337, 403.

Barrado y Navascués, D., Bouvier, J., Stauffer, J., Lodieu, N., \& McCaughrean, M. 2002, A\&A, accepted.

Bertelli, G. 2000, in "Stellar Clusters and Associations: Convection, Rotation, and Dynamos". R. Pallavicini, G. Micela and S. Sciortino (eds.) ASP Conf. Series 198, 115.

Chabrier, G., Baraffe, I., Allard, F., \& Hauschildt, P. 2000, ApJ, 542, L119.

Girardi, L., Bertelli, G., Bressan, A., Chiosi, C., M.A. Groenewegen, Marigo, P., Salasnich, B., \& Weiss, A. 2002, astroph 0205080.

Martín, E.L., Brandner, W., Bouvier, J., Luhman, K., Stauffer, J., Basri, G., Zapatero Osorio, M.R., Barrado y Navascués, D. 2000, ApJ, 543, 299.

Meynet, G., Mermilliod, J., \& Maeder, A. 1993, Navascués, D. 2000, ApJ, 543, 299. A\&AS98, 477.

Oke, J.B. et al. 1995, PASP, 107, 375.

de Zeeuw, T., Hoogerwerf, R., de Bruijne, J., Brown, A, and Blaauw, A. 1999, AJ, 117, 354. 